\title{
Contribution of Prof. Dr. Mahendra Pal in Veterinary and Medical Mycology
}

\author{
Pal M* \\ Director of Veterinary Public Health, India
}

*Corresponding author: Mahendra Pal, Founder Director of Narayan Consultancy on

Veterinary Public Health and Mycology, Anand, Gujarat, India; Email: palmahendra2@gmail.com

\section{Editorial}

Volume 2 Issue 2

Received Date: November 27, 2019

Published Date: December 19, 2019

DOI: $10.23880 /$ oajmms-16000110

\section{Editorial}

Mycology is a branch of microbiology, which deals with fungi that may affect the plants, animals, and humans. Fungi are eukaryotic, Gram positive, heterophilic, non-acid fast, saprobe and versatile group of living organisms, which are widely prevalent in our environment, and are isolated from soil, air, water, avian droppings, grains, fruits, vegetables, bagasse, woods, litter, and sewage etc.. [1]. Fungal infections occur in both sexes, in all age groups, in all seasons, in rural and urban settings, in all climatic zones, and in every strata of society. Some of the mycotic diseases, such as aspergillosis, candidosis, cryptococcosis, and zygomycosis can cause serious nosocomial outbreaks in newborn children, and immunocompromised patients [1].

It is predicted that approximately 5 million species of fungi are prevalent in the universe, of which only 600 have been found to be implicated with infections in humans and animals including birds [2]. In India, about 27,000 species of fungi are recorded by several researchers. It is stated that about $25 \%$ of world's cereals (food crops) are contaminated by fungal toxins annually. The loss of animal protein, such as milk, red meat, chicken meat, fish, and egg because of fungal infections, directly cause great economic losses to the dairy, fish, and poultry industries throughout the world. Mycotic diseases are important from public health as well as economic point of view, and are reported from developing and developed countries of the world. As mycoses are not modifiable, the correct data on their prevalence and incidence is not available. However, it is presumed that at least 800 million people in world have suffered from one or other types of fungal diseases indicating the growing significance of mycoses in human health [2].

Prof. Dr. Mahendra Pal is an internationally known scientist who worked in Veterinary and Medical Colleges in India and abroad, and published over 612 papers and authored 8 books. He has published papers on many fungal diseases, such as aspergillosis, candidiasis, coccidiodomycosis, cryptococcosis, dermatophytosis, fusariosis, geotrichosis, histoplasmosis, mycetoma, sporotrichosis, rhinosporidiosis, zygomycosis etc. His book entitled "Veterinary and Medical Mycology" is greatly appreciated by the academicians, and scientists throughout the world. Prof. Pal guided/advised over 67 students at DVM, MSc, MVSc, and Ph.D. level in India and Ethiopia. He has developed sunflower seed medium (Pal medium), APRM medium, PHOL stain, and Narayan stain for the studies of fungi implicated in many clinical disorders of humans and animals [1,3-5]. Prof. Pal is credited to elucidate for the first time the etiologic role of Cryptococcus neoformans in the mastitis of goat and buffalo, Trichophyton verrucosum in dermatitis of deer, Aspergillus niger in abortion of buffalo, Nocardia asteroides in keratitis of cattle, Aspergillus fumigates in ocular disorders of cattle and buffalo, Exserohium rostrum in equine dermatitis, Fusarium solani in corneal ulcer of buffalo, Aspergillus fumigates in rhinitis of camel and mule, Tricochosporon beigelii from the lung empyema of an immunocompromised patient and from burn wound of cattle, Curvularia lunata in keratitis of a rabbit, Aphanoascus fulvescens in canine dermatitis, and Candida tropicalis in human lung empyema. He has established for the First Time the prevalence of Cryptococcus neoformans 
in the environment of New Zealand, Nepal, and Djibouti. In addition, Prof. Pal has reported the first isolation of Candida albicans from mastitic milk, and Trichophyton verrucosum in dermatitis of camel in Ethiopia. He is recipient of several awards including Distinguished Teacher Award, Life Time Achievement Award, and International Achievement Award.

Prof. Pal work is frequently cited by many scientists in their papers, reviews, textbooks, and monographs. Many technologies such as APRM agar, sunflower seed medium (Pal medium), PHOL stain, and Narayan stain and the like developed by Prof. Pal, has greatly helped the academicians at national and international levels to progress in their research in the field of mycology. His more than 47 years continuous contributions to the role of fungi in animals and humans disorders have made an indelible mark on Veterinary Mycology in India and elsewhere. Due to his immense, outstanding and commendable contribution through several discoveries and achievements, Prof. Pal is considered the Father of Veterinary Mycology in India. His major mission in the life is to help the academicians to pursuit their research work in the field of Veterinary Mycology. Very recently, Prof. Pal founded "Narayan Consultancy on Mycology" to give free technical advice to the scientists of poor resource nations of the world. His more than 410 papers uploaded on international websites are viewed by 270,000 scientists of 170 countries of the world. Till now, over 2000 students are benefitted by the knowledge and wisdom of Prof. Pal.

Currently, mycotic diseases are recognized as significant causes of high morbidity and mortality in humans and animals throughout the world including India and hence should receive a greater attention at national and international levels. There is a need of multisectorial collaboration involving specialists from veterinary, medical, public health and wildlife in order to control mycoses. Moreover, further comprehensive and systematic work on the ecology, reservoir, transmission dynamics, and simple, sensitive and low cost diagnostics should be conducted. Sincere attempts should be made to develop safe, effective therapeutic agents to mitigate the morbidity and mortality due to most commonly encountered fungal diseases.

\section{Dedication}

The author wishes to dedicate this communication to all the scientists and academicians who worked for the development of veterinary and medical mycology.

\section{References}

1. Pal M (2007) Veterinary and Medical Mycology. $1^{\text {st }}$ (Edn.), Indian Council of Agricultural Research Institute, New Delhi, India.

2. Pal M (2018) Emerging role of saprobic fungi in human and animal health. Journal of Mycopathological Research 56(3): 1-2.

3. Pal M (1997) Use of Pal's sunflower seed medium for any early diagnosis of cryptococcosis. The Antiseptic 95(5): 175.

4. Pal M (2004) Efficacy of Narayan stain for morphological studies of moulds, yeasts and algae. Rev Iberoam Micol 21(4): 219.

5. Dave P, Pal M (2015) New medium "APRM" for isolation of medically important fungi from clinical and environmental samples. Int J Livest Res 5(3): 1018. 